\title{
SMART AGRICULTURE SYSTEM - A STUDY
}

\author{
Hemalatha K,Madhu manju,Navyashree N, Bhavana prabhu,Inchara V, Punitha M, Nethravathi B \\ Department of Information Science and Engineering \\ JSS academy of technical Education, Bangalore, Karnataka, India
}

\begin{abstract}
Agriculture in India has a vital role in the country's Economy. India's agriculture is composed of many crops and it is the backbone of our country. About 80\% of the population in India depending on farming. Hence the modernisation and automation of agriculture with techniques like Internet of Things has a major act in order to make it smart agriculture system. The Aim of project is to employ advance technology i.e. IOT and object detection techniques to come up with smart agriculture system using automation. Monitoring environmental conditions is the main factor to improve the yield of the efficient crops. With today's growing world and raising completion, the farmers are finding difficulty in monitoring the field and maintaining the crops. Hence, this project is developed to help the farmers to monitor the fields and protect and maintain the crops using the approach of Internet of Things (IOT) as well object detection techniques. The DC Pump is automatically switched ON/OFF by the Raspberry Pi and Relay, based on the soil moisture and the temperature level. Intrusion detection mechanism is used to protect the crop from animal or any theft. This is done by object detection technique which detects the type of animal attack and then notifies the farmer by an SMS and an image sent via mail to the farmer, so he can take the necessary steps to prevent higher extent of damage.
\end{abstract}

\section{Keywords - IOT (Internet of Things), DC (direct current)}

\section{INTRODUCTION}

Agriculture holds major parts of the Indian economy as it is main source of occupation in our country. Since farming is the means for food, agriculture can be considered as basis of human life, as well as animals. Also, it increases employment opportunities to the people. As per the statics study, agriculture employs over $50 \%$ of the Indian work force. Basically, Economic condition of our country depends on agriculture growth and its development. But even today, farmer still uses traditional methods for farming, which reduce in the yielding of crops. In traditional methods the resources required for farming cannot be utilized properly because of various reasons and lack of knowledge and more man work is required. To overcome this problem we need to automated machines and update our techniques. The main reasons for automation are to increase yielding of crop and reduce labor cost. So, it is necessary to use modern methods and technologies in agriculture sector for increasing crop growth and thus develop our economy.

One of the major problem farmer faces from everywhere is related to irrigation. Irrigation is method of supplying water to crops efficiently so that there will be improvement in food productivity. Water is one of the important things in farming, without water farmer will not have means of watering to their crops. Water and its management directly influence the crop growth. Most of the time, water cannot be used at proper time and there will be water wastage during irrigation. To fix this problem, we have to make use of an effective method for water management using technology Secondly, attack of birds and wild animals while growing or even when the the crop grows up is also an issue. There is always a prospect of robberies when the crops are at the point of harvesting. Even after harvesting of crops, farmers face problems in cold storages of harvested crop and monitoring the field also becomes a headache.

So, in order to offer clarifications to all such problems faced, it is essential to develop an integrated system which will be able to handle of all factors disturbing the productivity in every stages like; irrigation, harvesting, security, protection, etc. This project aims at coming up with Smart Farming system using automation and IOT technologies. Smart Farming is a farm management concept using modern technologies to increase the quantity and quality of agricultural yield. It's very essential to bring change in the way of advancement of agriculture. IOT based technology and it provides solution to it. Based on the calculation of agricultural parameters, we can have precision agriculture. It has played a vital role in the growth of agriculture in many developing countries. The introduction of wireless communication technology, sensors and CCTV cameras led to cost affective system. We propose a smart system with automatic irrigation system with automated water pump and also cameras and sensor based crop field monitoring and management system. It includes cameras which capture images at regular time period and image recognition techniques to recognize animal attack. 


\section{International Journal of Engineering Applied Sciences and Technology, 2020 Vol. 5, Issue 2, ISSN No. 2455-2143, Pages 439-444 \\ Published Online June 2020 in IJEAST (http://www.ijeast.com)}

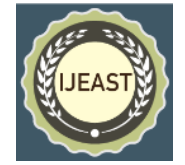

\section{RELATED WORK}

Existing agricultural research is being developed in different fields to improve the quality and quantity of production. Specialists have worked on many different projects on soil types, different climates and crop experiments. Christian University students worked on automatic irrigation using the IOT gate and animal penetration detection using motion sensors [1]. In addition to field monitoring The investigation of agriculture in area is developed in numerous parts in order to progress the quality and quantity of yield of farming. Students of Christ University worked on automatic irrigation using IOT gateway and also animal intrusion detection using motion sensors. The main aim is to predefine schedule of irrigation as per the farmer's accessibility, it's been uploaded into an application developed for the same. Here they mainly used automated irrigation powered by system photo-volts, That consists of different sensors which are immersed in a tree root Zones which are helped to monitor the soil and the environment parameters. This information is send into the IOT gateway which mainly consists of ARDUINO board. Wi-Fi module is installed in the IOT gateway which will be updating field parameter details to cloud. By using this gateway and GSM technology helps to guard the filed also in the absence of person by using android application. [2] In addition to monitoring the field here they have developed decision support advisory models which monitors the plants by image analysis which detecting the diseases and sends the warning to farmers using SMS based alerts. The advisory model consists of 3 main modules. Namely, Farm side, Client side and Server side. Farm side module consists of 3 layers namely precision, network and application layers. In farm field Ubi sense motes are installed in a different crop zones, Ubi is nothing but a sensor board which has some few sensor measuring the parameters like Light Intensity, Proximity sensing, temperature, Relative Humidity, Barometric Pressure, and Buzzer. The information collected in the UBI sense mote will be conveyed to server sideways. Based on this information the decision advisory model will alert the farmer. Client side module contains web applications and mobile applications for android devices.

[3] In this, smart automation irrigation system irrigates the crops field centered upon the data from the moisture sensor and temperature sensor in the fields whose value is sent to cloud database and turning on/off of the motor depends on the threshold value of temperature and moisture data saved in the cloud. Here they are used RaspberryPi 3. Python is used for software coding. Relay is used for automated irrigation. The main aim is to achieve high accuracy, data monitoring and monitor the automation system of agriculture with IOT Technologies. This system mainly concentrates on humidity deviations associated with changed value of temperature in sensors and to control irrigation system. To provide the cloud based computing for IoT system the accuracy level rises as suitable to use the system by agrarian.
Here in [4] IOT system architecture consists of four main parts: Controller, Memory, Wireless transceiver and Interface circuit. Controller part consists of microcontrollers in which input-signals is taken to perform control processes, according to the memory stored programs, and interrelate the decision made considering control-signals as output boundary. To insert required programs in to memory pogroming tools are used. The program is made using controller then it will be transferred into IOT sensor node. The memory unit comprises of programs that has to be performed will be stored. Input and output devices are used interface for the processor to receive information and communicate with the external devices. Here they implements distributed database or database tags, which consist of several data points. Data Points signifies one input or output to the system. It can be divided into 2 types, that is, it could be soft or hard, hard one signifies real input-output measured from devices where soft point signifies an arithmetic operations or a logic of the system. [5] This paper intends to develop a system which comprises two blocks and central computer to monitor. Each blocks contains various devices and sensors, they are furthers coupled with central pc through wire-less Zigbee modules. Through internet connectivity central pc sends and receives information from all the blocks. This system works generally in two modes; they are atomic and manual mode. In atomic mode, system can automatically monitor the field based on the measured parameters without taking the human intervention. In case of manual mode user has to operate the system using mobile apps or PC commands. Here they used GPS based vehicle system as first block, which is controlled remotely by central pc which comprises of different sensors like soil, obstacle, temperature, humidity etc. And collected data is sent to second block. Block 2 has the AVR microcontroller connected to the sensors of block 1 and depending up on the data received it automates the field. The third block consists of solar panels used to provide electricity for the whole system. This[6] paper introduces a concept of integrating image processing with IOT to give useful solution. The IOT sensor network captured the environmental factors. The changes in the plant due to temperature, humidity and other environmental factors are detected and analyzed through image processing using MATLAB software. By using IOT networks will do the process of capturing image and environment factors required for the plant. By using storage devices we can store the required data's for the process. In the section of image process, first step is by using camera image is clicked or captured then it is segmented by using method called $\mathrm{k}$ means clustering.

Using this method removes the noises that are oriented by different climatic condition and it is filtered then taken to next process. Processed image is taken to edge detection technique's, there are three edge detection techniques we are using in this process they are sobel, prewitt and canny algorithm. Among these techniques canny algorithm is one of the best results giving technology. And this is how to internet of things and image process can be used in agriculture to get 


\section{International Journal of Engineering Applied Sciences and Technology, 2020 \\ Vol. 5, Issue 2, ISSN No. 2455-2143, Pages 439-444 \\ Published Online June 2020 in IJEAST (http://www.ijeast.com)}

better result for crops.In paper [7] they have developed the system by using four modules. First modules is Data collection module and second one is Gateway Module, third module is Cloud Module and last module is end user module Data collection module: Temperature, humidity, pressure, intensity of light, $\mathrm{PH}$ value of soil, water level measurement for all these environment factors we need sensors, all these type of different kind of sensors are located in data collection module and also we placed set of cameras to capture images and videos at different interval of time. Gateway module: This is called as a connector because it connects the set of sensors and cameras placed in data collection module they are connected by wireless communication. Cloud Module: This module is data storage module, here data is collected from different sensors and catched data is compared with other data and analyzed for making decisions. After this decision making the information is sent to end user module. End user module will consists the farmer he will get the information by web or mobile application. In [8] In this paper deals with image processing by considering external management of crop. This is done by capturing image of plant at different interval of time and captured image is observed to know about morphological changes which will changes the development of crop. The conditions of plant like growth, dry, wet etc. This plant conditions information will be stored in cloud and it is shared through IOT to the end user devise like mobile or laptop. Addition to irrigation image processing is used to observe the health situation of the crops. The camera captures sample images and these images are analyzed with restored images in MATLAB software. This process is called as image acquisition. By using image smoothing technique, they remove noise in the images. This is called as Image Preprocessing. Images are segmented into parts and surface continuity and area perimeter are found. This is called as Image Segmentation. By analyzing predefined and sample image, the features are extracted and they conclude the outcomes. This is known as Feature Extraction.

In paper [9] in addition to automatic irrigation here they also concentrated on automatic seeding and spraying of the fertilizers. Farmers must maintain field periodically, so it is necessary to be automation. For the automation they have used aurdiuno and Ethernet shield. They use solar panel as power source which conserves the energy. Ethernet shield is used to provide internet connection for sending messages to external end user. They also make use of Blynk App to monitor the field conditions.

Here[10] the aspects of image process consist of acquisition, preprocess analysis and feature extraction. Image acquisition is concept of taking picture of plant as input by camera. And then it gets stored in database for next process. Preprocess of image includes removal of unwanted things of captured image and normalization is used in this aspect. And in image analysis captured image is segmented by using methods this will help to identify the healthy and unhealthy plants. Features extraction will consider the different features of plant like color, size, shape, texture. This will have the each and every information about the features. In addition to this, they have convolutional neural network to abstract tiny information about the image.

\section{PROPOSED METHODOLOGY}

Smart Farming System development using sensors and microcontrollers in the IOT system is developed here. The implementation of this project is to organize the smart and intellectual competencies of microcontrollers to water irrigate and to suggest plant varieties based on regular monitoring of environment conditions of the field. Intrusion detection can also be implemented using the CNN algorithm to protect crops from animal attack and theft. An electronic scarecrow is used to protect birds from damage to crops and effective crop monitoring is a farmer's concern for any other activity in the area.

As discussed earlier the Internet of Things system includes sensors and cameras for capturing the image of an animal, man and birds. The sensory network will capture various environmental conditions and send that information to the farmers using the trillio interface. Details according to the trillio information farmers can automatically tap on his or her pump. A sketch of the proposed block system is shown in Figure 1.

Here to optimize field conditions we use a humidity sensor, heat sensor, humidity sensor. Electricity is used to provide electricity throughout the mac cuit. Raspberry-pi is the primary control unit that connects all the IOT devices together to provide an IOT gateway. Dc water pump is used to irrigate the field. Camera will replace the filtered area when animals or theft enter the field. Touch motion detects movement of any bird or human-like obstacle is an animal and automatically sounds a buzzer. All IOT gate data is a suitable plant gateway based on soil and field conditions in a plant-ready file. -trillio. will help send field supplies to the farmers' phone.

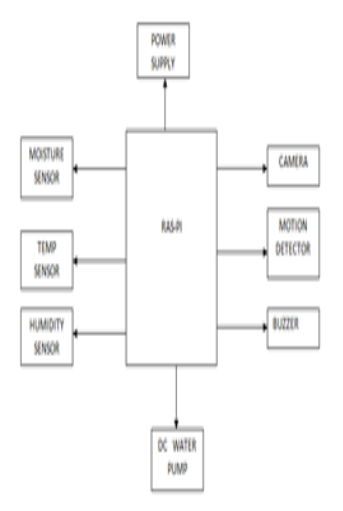




\section{International Journal of Engineering Applied Sciences and Technology, 2020 \\ Vol. 5, Issue 2, ISSN No. 2455-2143, Pages 439-444 \\ Published Online June 2020 in IJEAST (http://www.ijeast.com)}

Fig 1. Block diagram of smart farming.

The program works as a 2-module module on sensors and how they send agricultural conditions to farmers. The following module is based on image processing of the section. That is how the camera will take pictures of animals or any person who has entered the file. Here we have used the algorithm Convolution neural network that helps us classify the images captured by the camera mounted in the agricultural sector. The operating procedure of the Cnn will be shown in Figure 2.

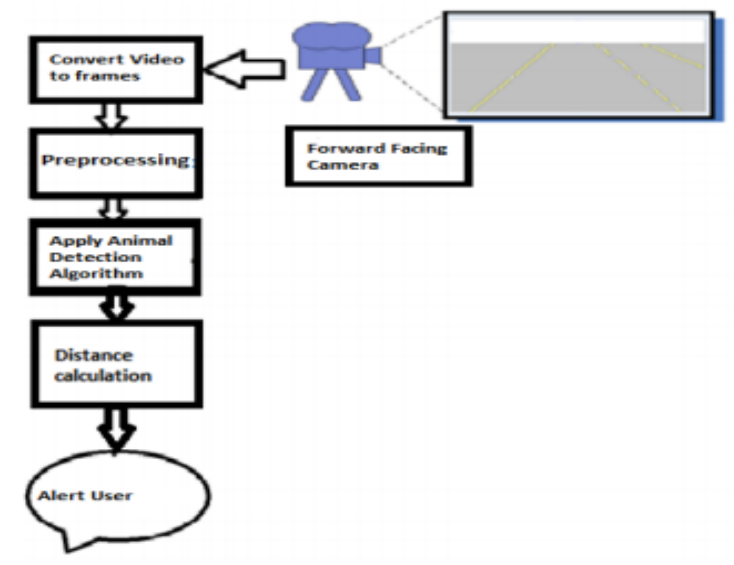

Fig 2. Measures of CNN Algorithm.

As we mentioned CNN Algorithms is basically used for image classification in intrusion detection. We developed a $\mathrm{CNN}$ model to detect the animals entered into the field. CNN works in 4 stages they are

- Image capturing: Camera is deployed in a farming field captures the live images of animals, birds or any person entered into the field.

- Image Analysis: Analysing the images and removal of noises in the image.In this phase we are looking for any motion detected in the live images, if the motion is detected that should be removed and the image will be organised as frames.

- Image pre-processing: Image pre-processing means extracting features in the input image, by using several methods like Gaussian distance formula, down sampling, Contrast enhancement that converting RGB images to grey scale images.

- Image classification and segmentation: In this phase, we are analysing the predicted value to pre stored data in a database. This gives the final prediction of the live image, about its name and accuracy of the image
Figure 3 represents the details of the IoT gateway connection. Transmission is automatically applied to the water pump. The heat sensor is connected to raspberry-pi through MCP-IC.

Since Raspberry-pi does not have an analog to digital converter the heat data recorded from the heat sensor it will be connected directly to the MCP-IC which converts the data from analog to digital and sends the data to raspberry-pi in a continuous process. MCP-IC is connected to Raspberry-pi pin 4.

Transmission is automatically applied to the water pump, connected to the Raspberry-pi pin 22. The humidity sensor is used to measure the natural humidity, which is connected to the number 3.The humidity sensor is used to measure the humidity in the soil connected to the Raspberry-pi pin number 21.

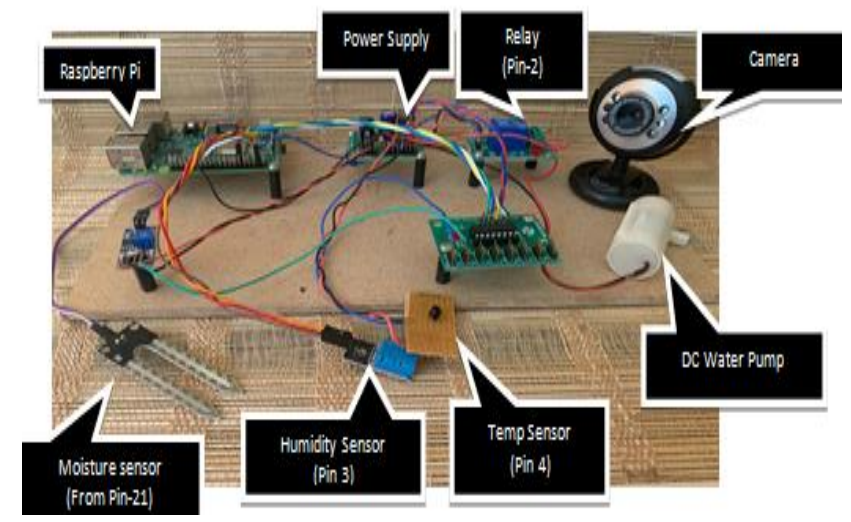

Fig 3. Circuit connection.

\section{Field Monitoring by Automatic Irrigation}

The core intention of this project is to monitor the field if any case farmer can't able go to his field every time in critical situation. In rural areas farm fields are situated from long distances from farmer house. So for this reason we developed an IOT network system, in that we deployed some of the sensors like moisture, temperature and humidity sensors are deployed in a farming field. These sensors read the environmental factors and they intern connected to RaspberryPi.

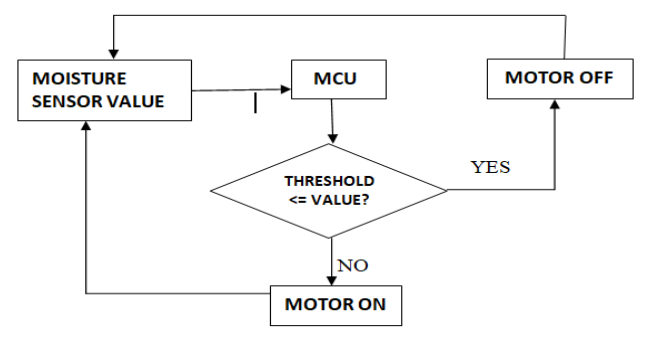

Fig 4. Turning on the water pump

Electronic Scare crow 


\section{International Journal of Engineering Applied Sciences and Technology, 2020 \\ Vol. 5, Issue 2, ISSN No. 2455-2143, Pages 439-444 \\ Published Online June 2020 in IJEAST (http://www.ijeast.com)}

Usually in large paddy fields the birds will harm the crops. Farmer makes some traditional methods of scaring the birds. But he can't able to watch the field from morning to evening. For that reason we have emanated with a smart sound system mechanism

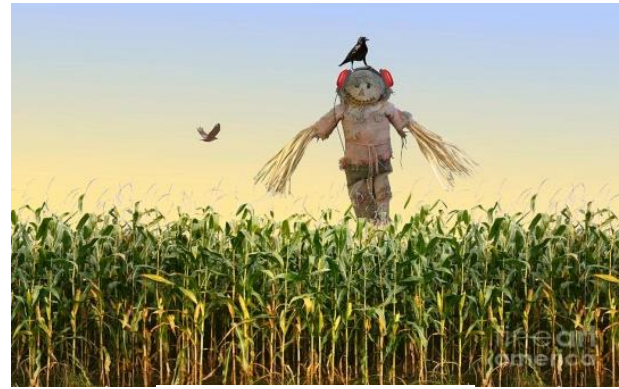

Fig.5.Scare crow setup

As discussed earlier, if any birds enters the field. Camera which is fixed in the field takes the pictures of birds. Whenever the birds are captured and detected by the camera, a python scripts used is interfaced with the sound system by turning on the buzzer sensor Buzzer produces a beeping or buzzing sound which scares the birds and to protect the crops.

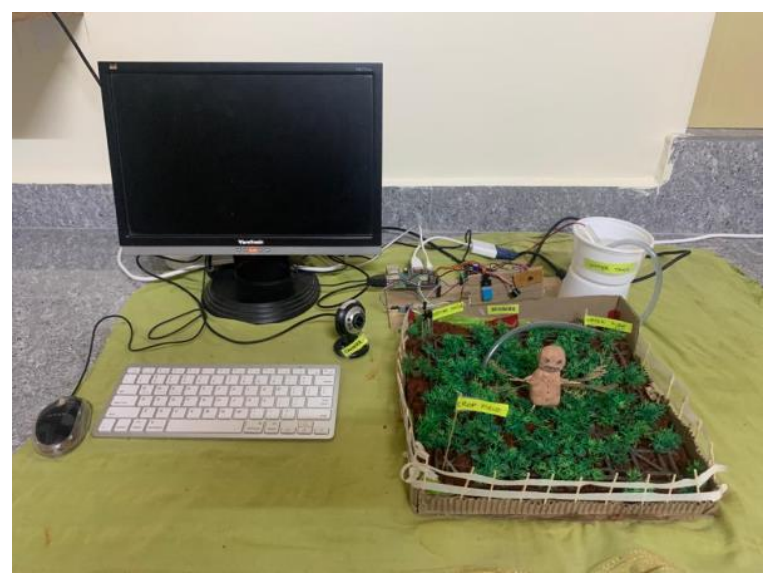

Fig.6. Smart farming system setup

\section{EXPERIMENT AND RESULT}

Eventually the object detector will detect animals by analyzing the images using the $\mathrm{CNN}$ algorithm that provides how much image detection was obtained. Calculates the accuracy and picture of the animal and the name of the animal is sent to the farmer. Figure 7 shows the effect of how $\mathrm{CNN}$ gives the result with respect to the intervention. After that he can make another decision. For example if an animal is wild you can take precautionary measures before entering the veld. Otherwise in case of small animals or birds, the buzzer will automatically open and frighten animals.

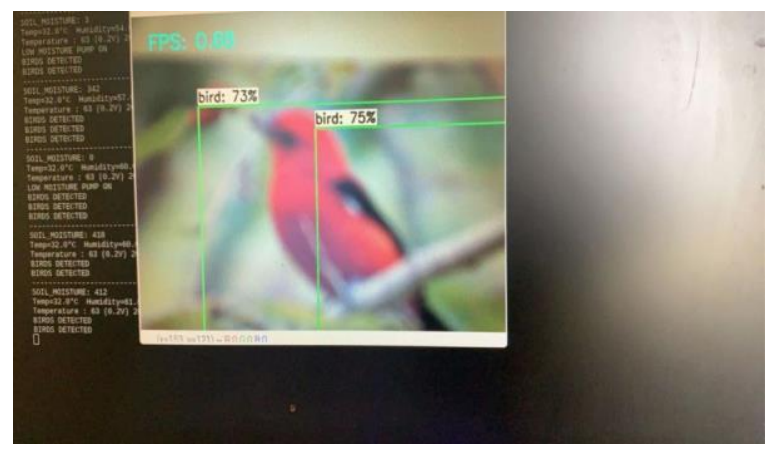

Fig 7a). Detection of birds

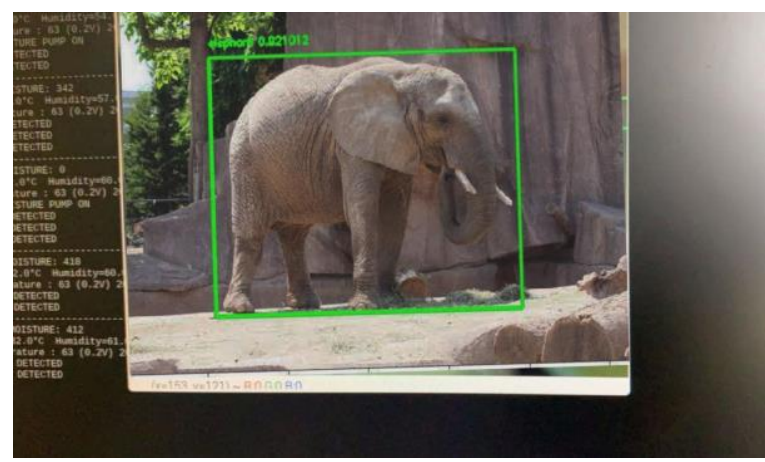

Fig.7.b). Detection of animals.

Fig 7.c) is snapshot taken from project which will detect animals in the field and sends alert messages to formers with detected animal name.

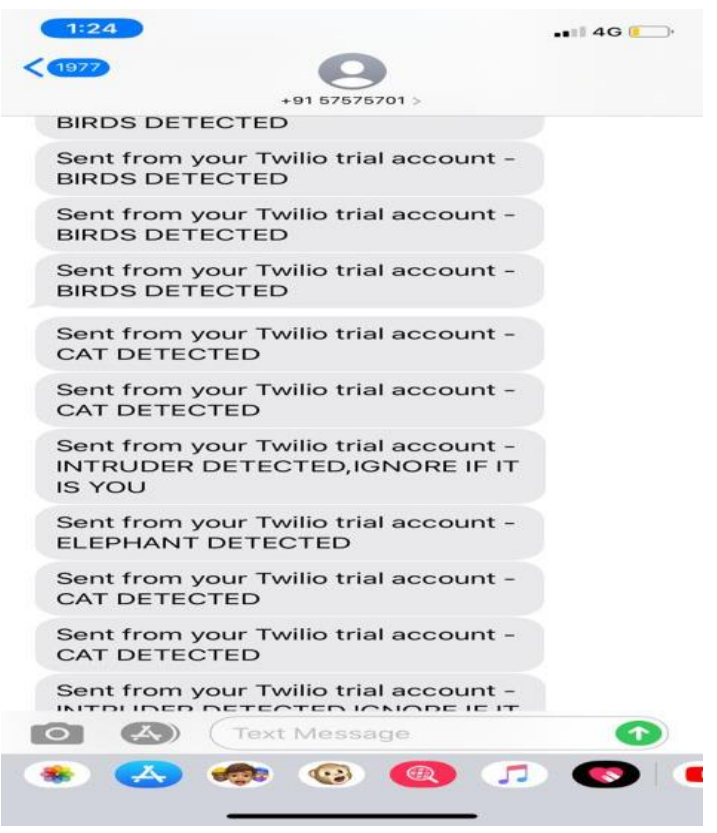

Fig.7.c). SMS alert and warning messages sent to farmer's mobile number 


\section{International Journal of Engineering Applied Sciences and Technology, 2020 Vol. 5, Issue 2, ISSN No. 2455-2143, Pages 439-444 \\ Published Online June 2020 in IJEAST (http://www.ijeast.com)}

\section{CONCLUSION}

For this project, we developed a Smart Agriculture Program. This is accomplished by doing simple tasks such as irrigation, field monitoring and crow shock. Improved accuracy in benchmark data related to other existing algorithms for animal recognition. Tests are accompanied on stationary pictures. In upcoming days, we are using the animal observation strategy on videos of animals and birds. In the future, we are working to develop an algorithm to increase precision. It can be kept and used under natural conditions to enhance any type of vegetation. Non-traditional energy sources such as solar panels can be used for additional applications. Agricultural input can be more efficient and commercially viable with additional IOT functionality. Plant protection creates a revolution in the way agriculture in India and the use of automated field rents

\section{REFERENCE}

[1] G. Sushanth and S. Sujatha, "IOT Based Smart Agriculture System", 978-1-5386-3624-4/18/\$31.00,IEEE 2018.

[2] Prof. K. A. Patil, Prof. N. R. Kale, "A Model for Smart Agriculture Using IoT",Information Computing and Communication, 978-1-5090-0467-6/16/\$31.00,IEEE 2016.

[3] R. Nageswara Rao and B.Sridhar, "IOT based crop monitoring and automation irrigation system", 978-15386-0807-4/18/\$31.00,IEEE (ICISC 2018).

[4] Amandeep, Arshia Bhattacharjee, Paboni Das Debjit Basu,Somudit Roy, Spandan Ghosh,Sayan Saha,'Smart farming using IOT", IEEE Conference on Open Systems (ICOS) 2018.

[5] K.Lakshmi and S.Gayathri, "Implementation of IoT with Image processing in plant growth monitoring system" , 978-1-5386-3371-7/17/\$31.00,JSIR 2017.

[6] Snowber Mushtaq," SMART AGRICULTURE SYSTEM + AND IMAGE PROCESSING", Volume 9, No. 1, January-February 2018, IJARCS.

[7] Rajendra Akhil Kumar, Gone Sri Vaishnavi, Pilli Sathavardhana Rao, Diwakar. R. Marur “ Crop Monitoring using Visual Sensors and IOT" SSRG IJECE,2017.

[8] Muthunoori Naresh and P Munaswamy "Smart Agriculture System using IoT Technology”, IJRTE ISSN: 2277-3878, Volume-7 Issue-5, January 2019.

[9] V R Balaji "Smart Irrigation System using Iot and Image Processing”,IJEAT ISSN: 2249 - 8958, Volume-8 Issue6S, August 2019.

[10] Ibrahim Mat, Mohamed Rawidean Mohd Kassim, Ahmad Nizar Harun, Ismail Mat Yusoff, "Smart agriculture system using iot" MIMOS Berhad, Kuala Lumpur, MALAYSIA.
[11] Yongxian Song, Juanli Ma, Xianjin Zhang, Yuan Feng, "Design ofWireless Sensor Network-Based Greenhouse Environment monitoring and Automatic Control System", JOURNAL OF NETWORKS, VOL. 7, NO. 5, MAY 2012.

[12] G.V.Satyanarayana and SD.Mazaruddin, "Wireless Sensor Based Remote Monitoring System for Agriculture using ZigBee and GPS", Conference on Advances in Communication and Control Systems 2013.

[13] D.Rajesh, "Application of Spatial Data Mining for Agriculture", International Journal of Computer Applications (0975-8887) Volume 15-No.2, February 2011.

[14] Dr. V .Vidya Devi,G. Meena Kumari, "Real- Time Automation and Monitoring System for Modernized Agriculture" ,International Journal of Review and Research in Applied Sciences and Engineering (IJRRASE) Vol3 No.1. PP 7-12, 2013.

[15] S. R. Nandurkar, V. R. Thool, R. C. Thool, "Design andDevelopment of Precision Agriculture System Using WirelessSensor Network", IEEE International Conference onAutomation, Control, Energy and Systems (ACES), 2014. 